\title{
Legibilidad de los documentos informativos en español dirigidos a lesionados medulares y accesibles por internet
}

\author{
Readability and internet accessibility of informative documents \\ for spinal cord injury patients in Spanish
}

\author{
M. Bea-Muñoz ${ }^{1}$, M. Medina-Sánchez ${ }^{2}$, M.T. Flórez-García ${ }^{3}$
}

\section{RESUMEN}

Fundamento. Los lesionados medulares y sus cuidadores tienen acceso en internet a folletos que pueden usar como complemento educativo frente a formas tradicionales de educación. El objetivo del estudio es valorar la legibilidad de los documentos informativos en español, obtenidos de internet y dirigidos a lesionados medulares.

Material y métodos. Se realizó una búsqueda en el buscador Google con las siguientes palabras clave: recomendación, consejo, guía, manual, autocuidado, educación e información, añadiendo lesión medular, paraplejia o tetraplejia con cada uno de los términos. Analizamos los primeras 50 resultados de cada búsqueda. La legibilidad de los folletos se estudió con los índices de Flesch-Szigriszt y el grado INFLESZ, ambos disponibles en el programa INFLESZ. También se indicó el año de publicación, el país y el número de autores de los documentos obtenidos.

Resultados. Se obtuvieron 16 documentos, elaborados entre 2001 y 2011. La legibilidad osciló entre 43,34 (algo difícil) y 62 (normal), con un valor medio de 51,56 (algo difícil). Solo 4 folletos (25\%) presentaron un índice de Flesch-Szigriszt $\geq 55$ (normal). No hay diferencias en la legibilidad según el año, los autores o el país de edición.

Conclusiones. La legibilidad del $75 \%$ de los documentos estudiados es "algo difícil" según la escala INFLESZ. Estos resultados coinciden con estudios previos, tanto en español como en inglés. Si conseguimos mejorar la legibilidad de este tipo de documentos, su objetivo educativo será más fácil de alcanzar.

Palabras clave. Lesión medular. Legibilidad. Folletos educativos. Internet.

An. Sist. Sanit. Navar. 2015; 38 (2): 255-262

\begin{abstract}
Background. Patients with spinal cord injuries and their carers have access to leaflets on Internet that they can use as educational material to complement traditional forms of education. The aim of this study is to evaluate the readability of informative documents in Spanish, obtained from Internet and aimed at patients with spinal cord injuries.
\end{abstract}

Methods. A search was made with the Google search engine using the following key words: recommendation, advice, guide, manual, self-care, education and information, adding spinal cord injury, paraplegia and tetraplegia to each of the terms. We analyzed the first 50 results of each search. The readability of the leaflets was studied with the Flesch-Szigriszt index and the INFLESZ scale, both available on the INFLESZ program. Also indicated were year of publication, country and number of authors of the documents obtained.

Results. We obtained 16 documents, developed between 2001 and 2011. Readability oscillated between 43.34 (somewhat difficult) and 62 (normal), with an average value of 51.56 (somewhat difficult). Only 4 pamphlets $(25 \%)$ showed a Flesch-Szigriszt index of $\geq$ 55 (normal). There was no difference in readability by year, authors or country of publication.

Conclusions. The readability of $75 \%$ of the documents studied was "somewhat difficult" according to the INFLESZ scale. These results coincide with previous studies, in both Spanish and English. If the readability of this type of documents is improved, it will be easier to achieve their educational goal.

Keywords. Spinal cord injury. Readability. Educational pamphlets. Internet.
1. Hospital Universitario Central de Asturias. Oviedo.

2. Catedrática de Fisioterapia. Universidad de Oviedo.

3. Jefe de la Unidad de Rehabilitación. Hospital Universitario Fundación Alcorcón. Madrid.

Recepción: 13 de noviembre de 2014

Aceptación provisional: 17 de diciembre de 2014

Aceptación definitiva: 24 de febrero de 2015

\section{Correspondencia:}

Manuel Bea Muñoz

Hospital Universitario Central de Asturias 33011. Oviedo. (España)

Email: mbeamedina@gmail.com

Los autores declaran que no han recibido ninguna beca o subvención para elaborar este trabajo. Tampoco tenemos relaciones financieras que puedan dar lugar a conflicto de intereses. 


\section{INTRODUCCIÓN}

Internet se ha convertido en una fuente de información habitual sobre problemas de salud $^{1-3}$. En 2013, el 44\% de los europeos buscó en internet información sobre la salud, un $6 \%$ más que en $2011^{4}$. Los datos de España coinciden con la media europea ${ }^{4} \mathrm{e}$ incluso, entre las personas de 22 a 55 años, Internet es la principal fuente de información sobre la salud en España, por delante del personal sanitario ${ }^{5}$. Aunque aún se observan claras diferencias sociales y entre países en el acceso a internet y en sus aplicaciones para la salud ${ }^{4,6}$, este uso de la red va a seguir creciendo en los próximos años ${ }^{6-7}$.

En los lesionados medulares las necesidades de educación e información sanitaria son muy elevadas. La lesión medular predispone a un gran número de complicaciones y problemas de salud, muchos de ellos prevenibles con una educación correcta $^{8}$. Además, para reforzar el principio bioético de la autonomía del paciente, para que éste tome sus propias decisiones, debe tener acceso a una información adecuada $^{9,10}$. Durante la rehabilitación hospitalaria el paciente se educa directamente con el personal sanitario, pero esta necesidad de formación debe mantenerse a lo largo de toda la vida ${ }^{11}$. Una buena alternativa para conseguirlo es utilizar folletos o documentos escritos que se entregan y se pueden consultar tras el alta hospitalaria. Los materiales impresos son económicos, sencillos de transportar y con contenidos fáciles de usar y mantener ${ }^{12}$. Sin embargo, para que la información contenida en estos documentos sea efectiva debe ser entendida por las personas a las que va destina$\mathrm{da}^{13}$.

Uno de los factores que más limitan la comprensión de un texto es su complejidad gramatical o legibilidad lingüística. La legibilidad es la facilidad con que se puede leer y comprender un texto. Depende en gran medida de si está constituido por palabras cortas y frases breves y de si su estructura permite al lector avanzar en el contenido del texto ${ }^{13,14}$. Las recomendaciones más importantes para mejorar la legibi- lidad de un texto se resumen en la tabla $1^{13}$. En Estados Unidos estas cuestiones han sido ampliamente estudiadas. El grado de Flesch-Kincaid, por ejemplo, indica el nivel de estudios que se necesita para comprender un texto ${ }^{15}$. El americano medio tiene un nivel de comprensión lectora de grado 8 según el sistema educativo de Estados Unidos (el equivalente a $2^{\circ}$ curso de Educación Secundaria en España), pero diversas instituciones americanas recomiendan que los textos de información sanitaria se escriban como máximo en un nivel de $6^{\circ}$ grado $\left(6^{\circ}\right.$ de Educación Primaria española $)^{15-18}$. Hay múltiples escalas para medir la legibilidad de un texto inglés: la fórmula de facilidad de lectura de Flesch, la ya mencionada de Flesch-Kinkaid, el índice de Gunning-Fog o el SMOG, entre otras. El sistema que utilizan es un recuento de palabras y sílabas por frase, aplicando después las fórmulas oportunas $^{14,19}$. La mayoría de las fórmulas de legibilidad parten de la hipótesis de que un texto es tanto más fácil de leer cuanto más cortas son las palabras y frases que utiliza $^{14}$. Sin embargo, estos instrumentos, creados para el idioma inglés, no se pueden aplicar sin más a textos españoles. Incluso, muchos de sus resultados se refieren a grados del sistema educativo anglosajón, limitando por tanto su interpretación en español. La fórmula de facilidad de lectura de Flesch ha tenido dos adaptaciones al español: la primera, por Fernández-Huertas en 1959 y una posterior, la realizada por Szigriszt-Pazos en 1993, considerada actualmente de referencia para esta escala ${ }^{20}$. En un trabajo posterior, Barrio-Cantalejo adaptó la escala de interpretación de resultados de Szigriszt-Pazos, creando la escala INFLESZ ${ }^{21}$. Estas escalas están disponibles en un programa informático gratuito, que facilita el análisis de la legibilidad de textos en español, accesible en la dirección web www.legibilidad.com.

Los estudios de legibilidad en inglés, tanto de folletos como de páginas web con información a pacientes suelen ofrecer unos resultados bastante pobres. La mayoría del material estudiado tiene una dificultad de lectura superior a la del lector medio y muy superior a los límites recomendados ${ }^{16,22-24}$. 
Tabla 1. Recomendaciones para mejorar la legibilidad de los folletos de educación para la salud.

\begin{tabular}{ll}
\hline Contenido y diseño del texto & 1) Estructurar el texto con títulos y subtítulos \\
& 2) Explicar bien el mensaje \\
& 3) Incluir conclusiones \\
\hline Construcción del texto & 1) Utilizar frases breves y simples. \\
& 2) Colocar las palabras con el orden más habitual: sujeto, verbo y complementos \\
& 3) Colocar la información más importante al principio de la frase \\
& 4) Evitar las construcciones pasivas y las negaciones \\
& 5) Incluir esquemas y ejemplos \\
6) Destacar las ideas principales
\end{tabular}

En español, los resultados no son muy diferentes. El programa INFLESZ se ha utilizado para analizar la legibilidad de un documento informativo para pacientes ${ }^{25}$, de diversos formularios de consentimiento informa$\mathrm{do}^{26-28}$ y de prospectos farmacéuticos ${ }^{29}$. No hemos encontrado ningún estudio que valore la legibilidad en documentos de información a lesionados medulares. Por tanto, el objetivo del presente estudio es conocer la legibilidad de los documentos, folletos y manuales de información dirigidos a lesionados medulares, en español y obtenidos mediante una búsqueda en internet.

\section{MATERIAL Y MÉTODOS}

Para identificar los documentos o folletos dirigidos a pacientes, hemos realizado varias búsquedas en Internet. El único motor de búsqueda utilizado fue Google España (www.google.es) ya que es el usado por más del $96 \%$ de los españoles ${ }^{30,31}$. Las búsquedas se realizaron entre el $14 \mathrm{y}$ el 16 de julio de 2014. Los términos utilizados fueron: recomendación, consejo, guía, manual, autocuidado, educación e información, añadiendo lesión medular, paraplejia o tetraplejia con cada uno de los términos, lo que da un total de 21 búsquedas. En cada una se analizaron los primeros 50 resultados (5 páginas de resultados de Google). Por tanto, el total de resultados revisados fue de 1050.

En cada página de las analizadas buscamos folletos, documentos o libros con información y contenido educativo para los lesionados medulares o sus cuidadores. Podían ser archivos pdf (Portable Document Format) o Word (Microsoft Office), descargables e imprimibles para su lectura posterior. Se consideró criterio de inclusión que los documentos fueran dirigidos específicamente a la información de lesionados medulares. Se descartaron por tanto los artículos científicos, los documentos dirigidos a profesionales o aquellos que estuvieran protegidos contra la copia de su contenido.

Una vez descargados los documentos, se analizó su legibilidad. Se utilizó el programa informático gratuito INFLESZ, disponible en la página www.legibilidad.com/ home/descargas.html. De forma automática el programa analiza el número de frases, palabras y sílabas, determinando el índice de Flesch-Szigriszt y el grado de dificultad de lectura en la escala INFLESZ. La fórmula utilizada fue $206,835-62,3 \mathrm{~S} / \mathrm{P}-\mathrm{P} / \mathrm{F}$, donde $\mathrm{S}$ es el número total de sílabas, $\mathrm{P}$ de palabras y $\mathrm{F}$ el número de frases del texto. Con la escala INFLESZ se considera que un texto tiene una legibilidad normal si puntúa entre 55 y 65 (Tabla 2). Previamente y para evitar errores en el análisis seguimos las recomendaciones del programa y preparamos el texto eliminando los saltos de página, los puntos en las direcciones web o en las abreviaturas y no se incluyeron en el análisis las citas bibliográficas. Todo esto lo realizamos copiando el contenido del folleto en un documento Word y apli- 
Tabla 2. Escala INFLESZ

\begin{tabular}{lcl}
\hline Grado de legibilidad & Puntos & \multicolumn{1}{c}{ Tipo de publicación equivalente } \\
\hline Muy difícil & $0-40$ & Universitario, Científico \\
\hline Algo difícil & $40-55$ & Bachillerato, Divulgación científica, Prensa especializada \\
\hline Normal & $55-65$ & Educación Secundaria, Prensa general, Prensa deportiva \\
\hline Bastante fácil & $65-80$ & Educación primaria, Prensa del corazón, Novelas de éxito \\
\hline Muy fácil & $>80$ & Educación primaria, Tebeos, Cómic \\
\hline
\end{tabular}

cando las herramientas automáticas "buscar" y "reemplazar" de dicho procesador de texto. Los documentos con menos de 5 apartados o capítulos se analizaron íntegros. Si tenían más, se escogieron al azar 5 capítulos mediante un generador virtual de números aleatorios sin repetición, disponible en la dirección web http://nosetup. org/php_on_line/numero_aleatorio_2. Tras analizar la legibilidad en esos 5 capítulos, elegimos la media aritmética de sus índices de Flesch-Szigriszt como medida global de legibilidad de ese documento. El análisis de la legibilidad lo realizó siempre el autor principal.

Se realizó un análisis descriptivo de la legibilidad global de los documentos según el índice de Flesch-Szigriszt indicando máximo, mínimo, media aritmética, desviación estándar e intervalo de confianza (IC) al $95 \%$. Además, se valoraron las siguientes variables:

- Año de publicación: Se diferenciaron dos grupos, las publicadas antes y después de 2007, buscando diferencias en la legibilidad de ambos grupos.

- Número de autores, separando las publicaciones realizadas entre 1 y 3 autores de las elaboradas por un colectivo mayor o por una institución.

- País de origen, comparando la legibilidad de los documentos españoles de los publicados en otros países.

Para el análisis estadístico de las variables indicadas se utilizó el test t de Student para variables independientes con un nivel de significación estadística de $\mathrm{p}<0,05$. El programa estadístico utilizado fue Me-
dCalc Statistical Software, versión 13.1.2 (MedCalc Software bvba, Ostend, Belgium; http://www.medcalc.org; 2014).

\section{RESULTADOS}

Tras la búsqueda en Internet se obtuvieron 16 documentos educativos o informativos dirigidos a lesionados medulares, en español y que cumplían los criterios de inclusión (Tabla 3). Sus años de publicación oscilan entre 2001 y 2011. Hay 9 documentos elaborados por un colectivo de autores o una institución y 7 realizados entre 1 y 3 autores. Respecto al país de edición, 6 documentos son españoles, 6 son traducciones de folletos de Estados Unidos y hay 2 argentinos, uno uruguayo y un documento venezolano.

Respecto a la legibilidad, el índice de Flesch-Szigriszt varía entre 43,34 y 62 , y su valor medio es de 51,56 (desviación estándar, 6,2416). Solo superan el punto de corte de 55 cuatro folletos, que se destacan en negrita en la tabla 3. Por tanto, solo estos 4 folletos (25\%) pueden considerarse con una legibilidad "normal" para el lector medio español. El resto se consideran "algo difíciles" según la escala INFLESZ.

No se observaron diferencias en la legibilidad de los documentos publicados antes o después de 2007 ( $\mathrm{p}=0,804$; IC 95\%, $-7,513$ a 9,449$)$. Tampoco hay diferencias al estudiar la legibilidad de los folletos realizados por 1-3 autores frente a los elaborados por un colectivo ( $\mathrm{p}=0,554$; IC $95 \%$, $-4,955$ a 8,841) ni al comparar la legibilidad de los publicados en España o en otros países ( $p=0,376$; IC 95\% -3,999 a 9,911). 
Tabla 3. Documentos analizados en el estudio, ordenados por fecha de edición

\begin{tabular}{|c|c|c|c|c|c|c|}
\hline Nombre & Url & Año & $\begin{array}{l}\text { I Flesch- } \\
\text { Szigriszt }\end{array}$ & $\begin{array}{l}\text { Grado } \\
\text { INFLESZ }\end{array}$ & Autores & Nacionalidad \\
\hline $\begin{array}{l}\text { Lesión medular. Guía de } \\
\text { autocuidados, } \mathrm{H} \mathrm{La} \mathrm{Fe}\end{array}$ & $\begin{array}{l}\text { www.san.gva.es/documents/246911/251004 } \\
\text { /V.5224-2005.pdf }\end{array}$ & 2001 & 46,71 & $\begin{array}{l}\text { Algo } \\
\text { difícil }\end{array}$ & Colectivo & España \\
\hline $\begin{array}{l}\text { La Lesión medular. Vejiga } \\
\text { e intestino }\end{array}$ & $\begin{array}{l}\text { www.minusval2000.com/pdf/la_lesion_medular_ } \\
\text { vejiga_intestino.pdf }\end{array}$ & 2002 & 44,29 & $\begin{array}{c}\text { Algo } \\
\text { difícil }\end{array}$ & Individual & España \\
\hline $\begin{array}{l}\text { A partir de ahora. } \\
\text { Orientación para } \\
\text { personas con lesión de } \\
\text { la médula espinal }\end{array}$ & $\begin{array}{l}\text { www.minusval2000.com/pdf/la_lesion_ } \\
\text { medular_vejiga_intestino.pdf }\end{array}$ & 2003 & 58,54 & Normal & Individual & Argentina \\
\hline $\begin{array}{l}\text { Úlceras por decúbito: lo } \\
\text { que usted debe saber }\end{array}$ & www.scicpg.org/cpg_span_pdf/PUC_span.pdf & 2004 & 62 & Normal & Colectivo & EEUU \\
\hline $\begin{array}{l}\text { Guía de Cuidados. HNP, } \\
\text { Toledo }\end{array}$ & $\begin{array}{l}\text { www.infomedula.org/images/stories/ } \\
\text { publicacionesLME/gua\%20cuidados\%20hnp.pdf }\end{array}$ & 2005 & 54,20 & $\begin{array}{l}\text { Algo } \\
\text { difícil }\end{array}$ & Colectivo & España \\
\hline $\begin{array}{l}\text { Lesión medular. Guía de } \\
\text { autocuidados, H La Fe }\end{array}$ & $\begin{array}{l}\text { www.san.gva.es/documents/246911/251004 } \\
\text { /V.5224-2005.pdf }\end{array}$ & 2006 & 48,30 & $\begin{array}{l}\text { Algo } \\
\text { difícil }\end{array}$ & Colectivo & España \\
\hline La vida sobre ruedas & $\begin{array}{l}\text { www.juntadeandalucia.es/export/drupaljda/ } \\
\text { La_vida_sobre_ruedas.pdf }\end{array}$ & 2007 & 61,43 & Normal & Individual & España \\
\hline $\begin{array}{l}\text { Lesiones de la médula } \\
\text { espinal: información para } \\
\text { personas con lesiones } \\
\text { recientes }\end{array}$ & $\begin{array}{l}\text { www.christopherreeve.org/atf/cf/\%7Bf94b00e6- } \\
\text { d099-4296-9544-1926c81d749a\%7D/NEW\%20 } \\
\text { INJURY\%20TOP\%20QUESTIONS\%209-08\%20_- } \\
\text { INTENATIONAL\%20SPANISH_\%202.11C.PDF }\end{array}$ & 2008 & 47,93 & $\begin{array}{l}\text { Algo } \\
\text { difícil }\end{array}$ & Colectivo & EEUU \\
\hline $\begin{array}{l}\text { Actividades de la } \\
\text { vida diaria. Manual } \\
\text { de enseñanza para el } \\
\text { paciente y la familia } \\
\text { sobre lesiones de la } \\
\text { médula espinal }\end{array}$ & $\begin{array}{l}\text { jdc.jefferson.edu/cgi/viewcontent. } \\
\text { cgi?article=1008\&context=spinalcordmanual_ } \\
\text { esp }\end{array}$ & 2009 & 60,64 & Normal & Colectivo & EEUU \\
\hline $\begin{array}{l}\text { Protocolo de abordaje } \\
\text { y tratamiento de } \\
\text { la sexualidad en el } \\
\text { lesionado medular }\end{array}$ & $\begin{array}{l}\text { http://www.bse.com.uy/wps/wcm/connect/ } \\
\text { ad248fa2-23fe-4b56-9356-573456ac6a3c/ } \\
\text { Protocolo_de_abordaje_y_tratamiento_de_ } \\
\text { la_sexualidad_en_el_lesionado_medular. } \\
\text { pdf?MOD=AJPERES\&CACHEID=ad248fa2-23fe- } \\
\text { 4b56-9356-573456ac6a3c }\end{array}$ & 2009 & 50,02 & $\begin{array}{l}\text { Algo } \\
\text { difícil }\end{array}$ & Individual & Uruguay \\
\hline $\begin{array}{l}\text { Lesión de la médula } \\
\text { espinal y rehabilitación } \\
\text { de la marcha }\end{array}$ & $\begin{array}{l}\text { www.msktc.org/lib/docs/SCI_Gait-Span_BZEdits. } \\
\text { pdf }\end{array}$ & 2011 & 51,36 & $\begin{array}{l}\text { Algo } \\
\text { difícil }\end{array}$ & Colectivo & EEUU \\
\hline $\begin{array}{l}\text { La espasticidad y las } \\
\text { lesiones de la médula } \\
\text { espinal }\end{array}$ & $\begin{array}{l}\text { www.msktc.org/lib/docs/SCI-spasticity-Span_ } \\
\text { BZEdits.pdf }\end{array}$ & 2011 & 48,47 & $\begin{array}{l}\text { Algo } \\
\text { difícil }\end{array}$ & Colectivo & EEUU \\
\hline $\begin{array}{l}\text { Fundación Christopher y } \\
\text { Dana Reeve. Lesión en la } \\
\text { médula espinal }\end{array}$ & $\begin{array}{l}\text { www.christopherreeve.org/atf/cf/\%7Bf94b00e6- } \\
\text { d099-4296-9544-1926c81d749a\%7D/SPINAL\%20 } \\
\text { CORD\%20INJURY_\%20LESION\%20DE\%20LA\%20 } \\
\text { MEDULA\%20ESPINAL\%202-11C.PDF }\end{array}$ & $\begin{array}{l}\text { No } \\
\text { consta }\end{array}$ & 52,11 & $\begin{array}{l}\text { Algo } \\
\text { difícil }\end{array}$ & Colectivo & EEUU \\
\hline $\begin{array}{l}\text { Consejos prácticos para } \\
\text { lesionados medulares }\end{array}$ & $\begin{array}{l}\text { www.discapacidadonline.com/wp-content/ } \\
\text { uploads/2011/06/consejos.practicos.para_. } \\
\text { lesionados.medulares.pdf }\end{array}$ & $\begin{array}{l}\text { No } \\
\text { consta }\end{array}$ & 51,81 & $\begin{array}{l}\text { Algo } \\
\text { difícil }\end{array}$ & Individual & Venezuela \\
\hline $\begin{array}{l}\text { Manual de educación } \\
\text { sanitaria para pacientes } \\
\text { lesionados medulares } \\
\text { con riesgo de padecer } \\
\text { úlceras por presión }\end{array}$ & $\begin{array}{l}\text { www.chubut.gov.ar/dpd/imagenes/Manual\%20 } \\
\text { prevencion\%20lesionados\%20medulares.pdf }\end{array}$ & $\begin{array}{l}\text { No } \\
\text { consta }\end{array}$ & 43,34 & $\begin{array}{l}\text { Algo } \\
\text { difícil }\end{array}$ & Individual & España \\
\hline $\begin{array}{l}\text { ¿Qué son y cómo se } \\
\text { pueden tratar los } \\
\text { músculos espásticos? }\end{array}$ & www.neurologiainba.com.ar/espasticidad.pdf & $\begin{array}{l}\text { No } \\
\text { consta }\end{array}$ & 43,83 & $\begin{array}{l}\text { Algo } \\
\text { difícil }\end{array}$ & Individual & Argentina \\
\hline
\end{tabular}

Nota: Los folletos con legibilidad normal se destacan en negrita. 


\section{DISCUSIÓN}

En nuestro estudio hemos identificado 16 documentos informativos en español, dirigidos a lesionados medulares. Nuestra intención no fue realizar una búsqueda sistemática y exhaustiva de folletos sino reproducir la búsqueda que realizaría en internet cualquier persona interesada. Revisar 5 páginas de Google nos parece suficiente ya que las búsquedas de la población general no suelen pasar de la segunda página de resultados, saltando de enlace en enlace ${ }^{32,33}$. En cuanto a los términos de la búsqueda, la mayoría de personas que consultan Internet sobre aspectos de salud utilizan un diagnóstico o síntoma como palabra clave ${ }^{5}$. En nuestra búsqueda, a los términos de lesión medular, paraplejia y tetraplejia hemos añadido las otras palabras clave ya descritas para obtener unos resultados más específicos.

Para el análisis de los documentos hemos seguido las pautas habituales de otros estudios. Si no se eliminan algunos signos de puntuación (punto y coma, dos puntos, guion), abreviaturas y decimales, se corre el riesgo de obtener una legibilidad más baja de lo que realmente es. También es necesario transformar todo el texto en un solo párrafo y eliminar los saltos de líneas ${ }^{15,18,34}$. La decisión de tomar 5 apartados o capítulos al azar para medir la legibilidad en documentos largos es similar a lo que hacen otros estudios al seleccionar un número de líneas o de palabras de un texto para determinar su legibilidad global ${ }^{15,35}$.

De los 16 documentos localizados, solo 4 son de lectura normal para el español medio, según la escala INFLESZ: dos son traducciones de material estadounidense, uno es argentino y otro español. No hay diferencias de legibilidad en los folletos según su fecha y país de publicación ni por el número de autores.

Hasta ahora no existían estudios de legibilidad de folletos en español dirigidos a lesionados medulares. Estudios previos de legibilidad en folletos para pacientes con otras patologías, en español, llegan a conclusiones similares, aunque usen la adaptación de Fernández-Huertas de la escala Flesch o analicen el material con las herramientas del procesador de texto Word 2000 de Microsoft ${ }^{36,37}$. Con las mismas escalas de nuestro trabajo se ha estudiado la legibilidad de diversos formularios de consentimiento informado y prospectos farmacéuticos españoles ${ }^{26-29}$. La mayoría de documentos son de lectura "algo difícil" en la escala INFLESZ. En los documentos de consentimiento informado ${ }^{28}$ hay diferencias significativas entre los de Urología (muy difíciles) y los de Angiología y Cirugía Vascular (normales). También se ha utilizado la escala INFLESZ para conseguir una legibilidad adecuada en un documento informativo para pacientes sobre adenoamigdalectomía $^{25}$. La legibilidad del material informativo para lesionados medulares se ha estudiado en inglés, con las escalas de Flesch y Flesch-Kincaid, analizando el contenido de varias páginas web de referencia ${ }^{24}$. El resultado indica que la mayoría de lectores americanos no comprenderían estos contenidos.

Son varias las limitaciones de nuestro trabajo. Primera, el carácter abierto y cambiante de Internet hace que búsquedas con otros motores o con otras palabras clave, pueden ofrecer resultados distintos. Sin embargo, el uso casi exclusivo de Google en España, los términos utilizados y la amplia búsqueda realizada hacen que nuestros resultados sean bastante fiables y similares a los que obtendría un ciudadano español haciendo una búsqueda de folletos en Internet. Segunda, no existe un patrón para medir la legibilidad ni en inglés ni en español. En inglés hay múltiples escalas. En nuestro estudio hemos usado la validación más reciente al español de la escala de Flesch, el índice de perspicuidad de Szigriszt-Pazos ${ }^{14,21}$.Tercera, reducir la capacidad para comprender un texto a una fórmula matemática también es objeto de críticas $^{34}$. La facilidad de lectura no solo depende de la arquitectura de las frases. Hay otros factores de difícil cuantificación como la complejidad del contenido, la puntuación del texto, la concentración del lector y sus conocimientos previos sobre el tema ${ }^{34,36}$. Aun así, las fórmulas de legibilidad nos aproximan a la dificultad teórica 
que puede tener el lector y nos ayudan a simplificar los textos para que sean más comprensibles $^{34,36}$. Por último, hay que recordar que nuestro estudio no ha entrado en la valoración del contenido de los documentos analizados.

Como conclusión, hemos encontrado 16 documentos de información a lesionados medulares en español, de origen y características diversas pero con una legibilidad "algo difícil" en el $75 \%$ de los casos. La gran necesidad de material educativo y de apoyo que precisan los lesionados medulares hace que tengamos que cuidar y mejorar la legibilidad de este tipo de documentos para pacientes y obtenidos en internet para facilitar su comprensión y así conseguir mejor su objetivo.

\section{BIBLIOGRAFÍA}

1. Los ciudadanos ante la e-Sanidad. Estudio sobre opiniones y expectativas de los ciudadanos sobre el uso y aplicación de las TIC en el ámbito sanitario. Observatorio Nacional de las Telecomunicaciones y de la Sociedad de la Información (ONTSI). [Consultado el 30 de agosto de 2014].Disponible en: http:// www.ontsi.red.es/ontsi/sites/default/files/ informe_ciudadanos_esanidad.pdf

2. Villaescusa Martínez V, Sáez Villar L. Búsqueda de información sobre salud a través de internet. Enferm Glob. 2013; 12: 197-205.

3. Marín-Torres V, Valverde Aliaga J, Sánchez Miró I, Sáenz del Castillo Vicente Mi, Polentinos-CasTRO E, GARRIDo BARRAL A. Internet como fuente de información sobre salud en pacientes de atención primaria y su influencia en la relación médico-paciente. Aten Primaria. 2013; 45: 46-53.

4. Individuos que usan internet para buscar información relacionada con la salud, 20072013. Observatorio Nacional de las Telecomunicaciones y de la Sociedad de la Información (ONTSI) [consultado el 30 de agosto de 2014]. Disponible en www.ontsi.red.es/ontsi/ es/node/334

5. Encuesta Pficer. El rol de Internet en el proceso de consulta de información sobre salud. Septiembre 2010. [consultado el 30 de agosto de 2014]. Disponible en www.pfizer. es/docs/pdf/noticias/Resultados_encuesta_ Pfizer.pdf

6. Higgins O, Sixsmith J, Barry MM, Domegan C. A literature review on health information- seeking behaviour on the web: a health consumer and health professional perspective. Stockholm: ECDC; 2011. [Consultado el 21 de abril de 2015]. Disponible en: http://ecdc.europa.eu/en/publications/Publications/Literature review on health information-seeking behaviour on the web.pdf

7. Kummervold PE, Wynn R. Health Information Accessed on the Internet: The Development in 5 European Countries. Int J Telemed Appl. 2012; 2012: 1-3.

8. Matter B, Feinberg M, Schomer K, Harniss M, BRown P, Johnson K. Information needs of people with spinal cord injuries. J Spinal Cord Med. 2009; 32: 545-54.

9. Beauchamp TL, Childress JF. Principios de Ética Biomédica,Barcelona: Masson, 1999.

10. GRacia Gulluén D. Procedimientos de decisión en ética clínica. $2^{\mathrm{a}}$ ed. Madrid: Triacastela, 2007.

11. MAY L, DAY R, WARREN S. Evaluation of patient education in spinal cord injury rehabilitation: Knowledge, problem-solving and perceived importance. Disabil Rehabil. 2006; 28: 405-13.

12. BRooks, BA. Using the Internet for Patient Education. Orthop Nurs. 2001; 20: 69-77.

13. Ferrando Belart V. La legibilidad: un factor fundamental para comprender un texto. Aten Prim. 2004; 34: 143-6.

14. ¿Que es la legibilidad? [Consultado el 30 de agosto de 2014]. Disponible en www.legibilidad.com/home/acercade.html\#legibilidad.

15. Sabharwal S, Badarudeen S, Unes Kunju S. Readability of Online Patient Education Materials From the AAOS Web Site. Clin Orthop Relat Res. 2008; 466: 1245-50.

16. Albright J, De Guzman C, Acebo P, Paiva D, FAULKNER M Swanson J. Readability of patient education materials: implications for clinical practice. Appl Nurs Res. 1996; 9: 139-143.

17. Greywoode J, Bluman E, Spiegel J, Boon M. Readability analysis of patient information on the American Academy of OtolaryngologyHead and Neck Surgery website. Otolaring Head Neck. 2009; 141: 555-8.

18. Shukla P, Sanghvi SP, Lelkes VM, Kumar A, Contractor S. Readability Assessment of Internet-based Patient Education Materials Related to Uterine Artery Embolization. J Vasc Interv Radiol. 2013; 24: 469-74.

19. Blanco Pérez A, Gutiérrez Couto U. Legibilidad de las páginas web sobre salud dirigidas a pacientes y lectores de la población general. Rev Esp Salud Publica. 2002; 76: 321-31.

20. GRÖNE O. Inventario de instrumentos para medir la legibilidad de un texto. [Consulta- 
do el 29 de diciembre de 2014]. Disponible en www.hospitalclinic.org/Portals/0/hospital\%20clinnic/corporaci\%C3\%B3/20091210In ventario_instrumentos_textoescrito.pdf

21. Barrio-Cantalejo IM, Simón-Lorda P, Melguizo M, Escalona I, MariJuán MI, Hernando P. Validación de la Escala INFLESZ para evaluar la legibilidad de los textos dirigidos a pacientes. An Sist Sanit Navar. 2008; 31: 135-52.

22. Ryhänen AM, Johansson K, Virtanen H, Salo S, SALANTERÄ S, LEINO-KILPi H. Evaluation of written patient educational materials in the field of diagnostic imaging. Radiography. 2009; 15:e1-e5.

23. BadarudeEn S, Sabharwal S. Assessing Readability of Patient Education Materials: Current Role in Orthopaedics. Clin Orthop Rel Res. 2010; 468: 2572-80.

24. Agarwal N, Hansberry DR, Singh PL, Heary RF, GolDSTEIn IM. Quality Assessment of Spinal Cord Injury Patient Education Resources: Spine. 2014; 39:E701-E704.

25. Escudero-Carretero MJ, Sánchez-Gómez S, González-PÉrez R, Sanz-Amores R, Prieto-Rodríguez MA, FERnÁNDEZ dE LA MotA, E. Elaboración y validación de un documento informativo sobre adeno-amigdalectomía para pacientes. An Sist Sanit Navar. 2013; 36: 21-33.

26. Álvarez-Díaz JA. Legibilidad de los formularios de educación y consentimiento en procedimientos de reproducción asistida de la Red Latinoamericana de Reproducción Asistida. Cir Cir. 2012; 80: 162-70.

27. Ramírez-Puerta MR, Fernández-Fernández R, FríasPareja JC, Yuste-Ossorio ME, Narbona-Galdó S, Peñas-Maldonado L. Análisis de legibilidad de consentimientos informados en cuidados intensivos. Med Intensiva. 2013; 37: 503-9.

28. San Norberto EM, Gomez-Alonso D, Trigueros JM, Quiroga J, Gualis J, Vaquero C. Legibilidad del consentimiento informado quirúrgico en España. Cir Esp. 2014; 92: 201-7.

29. Ballesteros-Peña S, Fernández-Aedo I. Análisis de la legibilidad lingüística de los prospectos de los medicamentos mediante el índice de Flesch-Szigriszt y la escala INFLESZ. An Sist Sanit Navar. 2013; 36: 397-406.

30. Search Engine Barometer, July 2014. The search engine market in France, Germany, Spain and United Kingdom. [Consultado el 30 de agosto de 2014]. Disponible en: http:// www.atinternet.com/en/documents/searchengine-barometer-july-2014/

31. ¿Cuáles son los navegadores y buscadores que más se utilizan en el mundo? [Consultado el 30 de agosto de 2014]. Disponible en: http://www.webyposicionamientoseo.com/ cuales-son-los-navegadores-y-buscadoresque-mas-se-utilizan-en-el-mundo.blog

32. Eysenbach G, KöHLeR C. How do consumers search for and appraise health information on the world wide web? Qualitative study using focus groups, usability tests, and indepth interviews. BMJ 2002; 324: 573-7.

33. Mira Solves JJ, Llinas Santacreu G, Lorenzo MarTíNEz S, PÉREZ-Jover V. Preguntas más frecuentes sobre repercusión de la e-salud en la relación entre médico y paciente. Aten Primaria. 2010; 42: 112-4.

34. Friedman DB, Hoffman-Goetz L. A Systematic Review of Readability and Comprehension Instruments Used for Print and Web-Based Cancer Information. Health Educ Behav. 2006; 33: 352-73.

35. Barrio Cantalejo I, Simón Lorda P. Medición de la legibilidad de textos escritos. Correlación entre método manual de Flesch y métodos informáticos. Aten Primaria. 2003; 31: 104-8.

36. Ávila de Tomás JF, Veiga Paulet JA. Legibilidad de la información sanitaria ofrecida a los ciudadanos. Una aproximación a través del Índice de Flesch. Centro de salud. 2002; 10: 589-97.

37. Barrio Cantalejo I, Simón Lorda P ¿Pueden leer los pacientes lo que pretendemos que lean? Un análisis de la legibilidad de materiales escritos de educación para la salud. Aten Primaria. 2003; 31: 409-14. 